\title{
Review of: "The Impact of Intra-Uterine Manipulators on Outcome and Recurrence Patterns of Endometrial Cancer Patients Undergoing Minimally Invasive Surgery"
}

\author{
Jianliu Wang
}

Potential competing interests: The author(s) declared that no potential competing interests exist.

This is a very interesting and informative study regarding the oncological safety of uterine manipulator in endometrial cancer surgery. The results provide some new insights into understanding the potential risks of using uterine manipulator and the safety of minimally invasive surgery in endometrial cancer treatment. And I believe, this topic adheres to the interest of the gynecologic oncology community, especially after the publication of the LACC trail data. However, there are several aspects that need to be further revised before publication.

1. In the method - study population and setting section, since the author mentions that "All surgeries were performed by laparoscopic or robotic approach by 6 gynecologic oncology surgeons", more descriptions are needed regarding the qualifications and surgical experience of these surgeons. Namely, why these 6 doctors were chosen in the study? Was the inter-case consistency guaranteed? Did the surgical manipulations follow the same procedure so as to be comparable? These questions need to be settled.

2. It would be better to provide the photos and detailed parameters about the uterine manipulators used in the supplementary material.

3. In the clinical information section, the definition of OS is potentially problematic because the time from diagnosis to last follow-up should not be OS and such cases are censored at their last follow-up in survival analysis.

4. In statistical analysis section, for Cox regressions, the proportional hazard hypothesis should be tested. And the method for it should be included in this part.

5. In the results section, since EIN and endometrial cancer are different diseases, and this study is about EC, it is redundant to firstly include EIN and then exclude it. To address the inclusion criteria for EC cases is enough. And it is better to put the inclusion criteria in the method section. Besides, is there any patient with concurrent or history of other tumors? Such cases may introduce bias in survival analysis. This should also be addressed.

6. The indications for PLND, PALND and SLND should be included. And risk factors based on which adjuvant treatment is delivered should also be mentioned.

7. As is shown in the table, the baseline characteristics of the intra-uterine manipulator group and no intra- 
uterine manipulator group are different in terms of many clinicopathological factors. This may introduce potential bias for survival analysis. Propensity score matching or IPTW methods could be used here for adjusting it.

8. The author conducted multivariate Cox regression. But the inclusion criteria for clinicopathological factors included in the model (namely, which factor should be included and why) is not mentioned. This should be addressed for better assessing the rationality of the model.

9. In the discussion section, the author mentions that "We can speculate that the effect of higher recurrence rates on long term outcome of patients in the manipulator group, was eventually diluted by additional treatment modalities and that the presence of a uterine manipulator during surgery did not negatively affect the outcome of endometrial cancer patients on multivariate analysis." Actually, as a method of controlling confounding bias, multivariate analysis is a method to reveal the independent influence of each variable. In this sense, the survival influence of uterine manipulator should be belied in univariate analysis, due to the confounding of adjuvant treatment, and should be revealed in multivariate analysis. It seems that the author misconstrued the logic.

10. In page 14, on the second line from the bottom, it should be "which is in agreement with previously published data".

11. In the same paragraph, the author mentions that "the use of intrauterine manipulator was not associated with a significant difference in the rate of LVSI". The author listed related study results, but didn't provide an analysis of the reasons for the results. An analysis should be added in this part.

12. An analysis of future directions of research should also be provided in the discussion section. 